\title{
Nationalsozialistische und protofaschistische Sympathien an der Universität Freiburg i. Ü.: Die Ausweisung von Josef Spieler und die Neubesetzung des Lehrstuhls für Heilpädagogik durch Eduard Montalta
}

\author{
Daniel KÜNZLER und Christoph TSCHANZ1
}

Universität Fribourg

\begin{abstract}
Einleitung
Anlass zu diesem Text ist das Befremden über eine Kurzbiographie über Josef Spieler, welche 2004 in der heilpaedagogik.de veröffentlicht wurde (Berger 2004). Josef Spieler (geb. 1900 in Walldürn (Baden-Württemberg), gest. 1987 in Karlsruhe) hatte an der Universität Freiburg im Üechtland in der Schweiz von 1933 bis 1945 eine Professur in Psychologie, Pädagogik und Heilpädagogik inne. Diese wurde ihm 1945 entzogen und er musste aufgrund nationalsozialistischer Sympathien 1947 die Schweiz verlassen. Manfred Berger beschreibt in dieser Biographie in einer durchaus positiven und anerkennenden Art und Weise das heilpädagogische Schaffen Josef Spielers in der Schweiz und, nach seiner Rückkehr 1947, in Deutschland. Die Kurzbiographie befremdet insbesondere, weil sie nicht erwähnt, dass Spielers Rückkehr nach Deutschland nicht freiwillig erfolgte. Bereits in den frühen 1990er-Jahren wird in der Literatur thematisiert, dass Spieler wegen seiner nationalsozialistischen Sympathien der Lehrstuhl entzogen und er aus der Schweiz verwiesen wurde (vgl. Haeberlin 1990: 331-332; Raab 1991: 304; Oser 1991: 761; Haeberlin 1991: 763). Zu Beginn der 2000er-Jahre hatte auch die historische Aufarbeitung der Verbindung von Spieler mit dem Nationalsozialismus bedeutende Fortschritte gemacht (Schweizer 2002; Wolfisberg 2002). Die Kurzbiographie von Berger (2004) bleibt damit in problematischer Weise hinter dem damals vorhandenen Forschungsstand zurück. Manfred Berger ist anzurechnen, dass er in einem sechs Jahre später geschriebenen Kirchenlexikoneintrag den Forschungsstand adäquater abbildet und auf verschiedene der genannten Arbeiten Bezug nimmt (Berger 2010). Er relativiert allerdings darin die erzwungene Ausweisung erneut.

In der Zwischenzeit hat das Internationale Archiv für Heilpädagogik die online verfügbare Biographie von Manfred Berger durch einen kurzen Text der beiden Autoren ergänzt (Tschanz und Künzler 2018). Wir möchten an dieser Stelle den aktuellen Forschungsstand zu

\footnotetext{
${ }^{1}$ Daniel Künzler ist Lektor am Departement für Sozialarbeit, Sozialpolitik und globale Entwicklung (Fribourg) und vertritt zurzeit die Professur für Entwicklungssoziologie an der Universität Bayreuth: daniel.kuenzler@unifr.ch; Christoph Tschanz ist Doktorand SNF am Departement für Sozialarbeit, Sozialpolitik und globale Entwicklung (Fribourg): $\underline{\text { chris- }}$ toph.tschanz@unifr.ch. Die Aufzählung der Autoren ist alphabetisch. Beide haben zu gleichen Teilen zum Manuskript beigetragen.
}

DOI: http://dx.doi.org/10.18753/2297-8224-134 
Spielers Zeit in der Schweiz etwas ausführlicher darstellen und auf Forschungslücken hinweisen. Forschungslücken gibt es auch im Hinblick auf die anschliessend diskutierte Frage, wie es Spieler gelang, nach seiner Rückkehr nach Deutschland wieder im Universitätssystem Fuss zu fassen. Hierbei war ein Netzwerk um die Caritas zentral, welches die Universitäten in Freiburg im Üechtland und in Freiburg im Breisgau miteinander verband. Abschliessend möchten wir auf eine weitere Forschungslücke verweisen: Zur lückenhaften Aufarbeitung nationalsozialistischer Sympathien an der Universität Freiburg i. Ü. gehört, dass auch im Werk von Spielers schweizerischem Nachfolger Eduard Montalta durchaus problematische Positionen vertreten wurden.

\section{Forschungsstand zur Ausweisung von Josef Spieler}

Kurz vor Ende des 2. Weltkrieges hat die explizit antifaschistische Zeitung "Nation“ in einem Artikel Spieler stark belastet und dem später abgewählten freiburgischen Erziehungsdirektor Josef Piller vorgeworfen, Spieler und andere Professoren mit Sympathien für den Nationalsozialismus zu decken (vgl. Wolfisberg 2002: 125; Dorand 2017: 42). Dies hatte zur Folge, dass die Bundesanwaltschaft tätig wurde und der schweizerische Bundesrat am 20. Juli 1945 die Ausweisung von Spieler wegen "Gefährdung der inneren und äusseren Sicherheit" verfügte (Wolfisberg 2002: 128). Nach mehreren Wiedererwägungsgesuchen wurde die Ausweisungsverfügung am 21. März 1947 vom Bundesrat letztmalig bestätigt und eine Ausreisefrist auf den 20. Mai 1947 angesetzt, welcher Josef Spieler mit der Übersiedlung nach Freiburg i. Br. nachgekommen ist (Wolfisberg 2002: 134).

Der Entscheid basierte einerseits auf drei nachgewiesenen Anschuldigungen. Erstens konnte die Bundesanwaltschaft beweisen, dass Josef Spieler seit dem 1. Februar 1940 Mitglied der NSDAP war (Wolfisberg 2002: 133). Als Verteidigung trug Spieler vor, dass er in der deutschen NSDAP nur Mitläufer war und dort als unzuverlässig galt. Diese Aussage wurde von Karl Tornow mit einer eidesstattlichen Erklärung unterstützt (Wolfisberg 2002: 133). Tornow, mit dem Spieler freundschaftlich verbunden war (Eberle 2010), war vor Kriegsende Mitarbeiter im Rassenpolitischen Amt der NSDAP (Hänsel 2009: 782). Zweitens war beweisbar, dass Josef Spieler ab Februar 1943 vom Deutschen Reich monatlich mit 120 Reichsmark unterstützt wurde (Wolfisberg 2002: 124). Drittens wurde Spieler durch das Verdienstkreuz der II. Klasse belastet, welches er im März 1945 von der deutschen Botschaft erhalten hatte (vgl. Raab 1991: 304-305, Wolfisberg 2002: 131). ${ }^{2}$ Der Anlass für die Verleihung des Verdienstkreuzes wird in der Literatur nicht genannt.

Da Spieler wegen verdächtigen Aktivitäten ab 1939 polizeilich und nachrichtendienstlich überwacht wurde, lagen ausserdem belastende Beobachtungen vor (Wolfisberg 2002: 123-124). Dokumentiert sind zahlreiche Besuche von Veranstaltungen der NSDAP-Ortsgruppe Luzern, im deutschen Amtsbureau und Treffen mit anderen von der Bundesanwaltschaft verdächtigten Personen (Wolfisberg 2002: 124). Spieler soll im Jahre 1943 zudem zwecks Spionage eine

\footnotetext{
${ }^{2}$ Ebenfalls ausgezeichnet und danach des Landes verwiesen wurden drei andere Professoren der Universität Freiburg i.Ü. (Richard Newald, Heribert Reiners und Emil Tonutti). Freiwillig die Schweiz verlassen hat der Professor für Zoologie und zeitweiliger Stützpunktleiter der NSDAP, Hubert Erhard.
} 
Affäre mit einer Mitarbeiterin der schweizerischen Zentralstelle für Kriegswirtschaft gehabt haben (Wolfisberg 2002: 124).

Der Grossteil der gegen Josef Spieler vorgetragenen Beschuldigungen muss als Gerüchte und persönliche Anschuldigungen bezeichnet werden, die nie gerichtlich beurteilt wurden. Dazu gehören Sympathien für die frontistische Bewegung, sowie deutschfreundliche und mehrmalige positive Äusserungen über Rassenlehre und Sterilisationen (Wolfisberg 2002: 123127). Zeuge für zahlreiche persönliche Anschuldigungen gegen Spieler war Doktorand Späni, dessen explizit anti-nationalsozialistische theologische Dissertation durch die Professoren Spieler, Newald und Reiners im Jahre 1942 nicht angenommen wurde (Wolfisberg 2002: 130). Späni warf Spieler etwa vor, in seinen Vorlesungen nationalsozialistisches Gedankengut zu vermitteln und bei Exkursionen in informeller Umgebung unter Alkoholeinfluss sehr explizit nationalsozialistische Äusserungen getätigt zu haben.

\section{Forschungsstand zu den Tätigkeiten und Publikationen von Josef Spieler}

$\mathrm{Zu}$ den Inhalten der universitären Lehrveranstaltungen Spielers fehlen bislang systematische Forschungen. Auch seine gleichzeitige praktische heilpädagogische Arbeit als Direktor des Instituts für Heilpädagogik und als Leiter der heilpädagogischen Beobachtungsstationen in Wangen bei Olten (SO) und Knutwil (LU) sind bislang nicht erforscht. Diese scheint fast wichtiger zu sein als die akademische Arbeit in Freiburg i. Ü. (Wolfisberg 2002: 116), im Laufe derer er bemerkenswerterweise nicht $\mathrm{zu}$ heilpädagogischen Fragen publizierte, sondern hauptsächlich im Bereich Erziehung und Familienberatung. Für Spielers Verhältnis zum Nationalsozialismus sind vor allem drei Arbeiten interessant, die in seiner Schriftenreihe erschienen sind.

Die erste ist die von Spieler betreute Dissertation des deutschen Doktoranden Othmar Englert „Die Abnormenzählungen in Deutschland und in der Schweiz“. Die sozialdarwinistische Arbeit stellt die nationalsozialistische Rassenhygiene in Deutschland als Vorbild für die Schweiz und als pädagogische Aufgabe dar. In seinem Vorwort bedauert Spieler (in Englert 1942), dass die eigentliche Absicht der Dissertation, nämlich die praktische Durchführung einer ersten „Abnormenzählung“ in der Schweiz, „leider durch die politischen Verhältnisse jäh unterbrochen wurde“. Englert war nach seiner Rückkehr nach Deutschland unter anderem in der Abteilung „Erb- und Rassenpflege“ des Gesundheitsamtes der Stadt Magdeburg mit der „erbbiologischen Erfassung“ von sogenannten asozialen Familien beschäftigt (Englert 1942: 103).

Spieler gab auch die von ihm betreute Dissertation von Alois Gügler zur „erziehlichen Behandlung jugendlicher männlicher Onanisten“ heraus. Spieler fokussierte sich in seinem Vorwort (in Gügler 1942) auf das Argument der Schwächung der Volkskraft durch die Onanie: „Nicht schwächliches Gewährenlassen und Nachgiebigkeit, sondern nur Heroismus und heldenhafte Ueberwindung befreit den Einzelnen, bewahrt die Zukunft der Familie, stärkt ein Volk im Dienst am ganzen Menschengeschlecht." Bei der Einreichung der Dissertation war Gügler bei der Caritaszentrale in Luzern angestellt, später zur Entlastung von Spieler am Institut für Heilpädagogik in Luzern beschäftigt (Marti 1997: 32). 
Spieler gab auch die Arbeit von Faustin Pittet zur „Stellung der Kirche zur Eugenik und Rassenhygiene" heraus. Spieler schrieb nur ein kurzes Vorwort (in Pittet 1942), in dem er die „kurzen, prägnanten Formulierungen“ als „klare Wegweisung“ für Katholiken bezeichnete. Nach Pittet (1942: 20) teile die katholische Kirche das Ziel der Volksgesundheit und leiste durch ihre Haltung zu Ehe und Familie „wertvollste [positive] Eugenik“, lehne aber die „negative Eugenik“, also Massnahmen wie Euthanasie oder Zwangssterilisationen, ab.

Insgesamt kann Wolfisberg (2002: 136) zugestimmt werden: Spieler war ein „überzeugter Katholik und auch recht offen gegenüber dem Nationalsozialismus“; jedenfalls „offener, als er aus rein taktischen Gründen hätte sein müssen“.

\section{Spielers Rückkehr nach Deutschland und das Netzwerk um die Caritas}

Wie konnte es Spieler gelingen, nach seiner Rückkehr nach Deutschland im Universitätssystem wieder Fuss zu fassen? Diese Frage ist unseres Wissens nur sehr lückenhaft aufgearbeitet. Spieler wurde vom späteren Leiter des Instituts für Caritaswissenschaften an der Universität Freiburg i. Br., dem Theologen und Heilpädagogen Linus Bopp, 1947 erfolglos für die Besetzung einer pädagogischen Professur an dieser Universität vorgeschlagen mit dem Hinweis, "politische Schwierigkeiten seien nicht zu erwarten“ (Kersting 2008: 295). Spieler erhielt im Wintersemester 1948/49 lediglich Lehraufträge. Er wurde 1955 vom Akademischen Rektorat der Universität Tübingen erfolglos für die Wiederbesetzung des ordentlichen Lehrstuhls für Pädagogik und Philosophie ins Spiel gebracht (Kersting 2008: 249). Nach einer Professur in Weilburg an der Lahn (Hessen) wurde er dann aber ab 1952 bis zu seiner Emeritierung 1965 Professor und Rektor am Pädagogischen Institut Karlsruhe, welches 1962 zur Pädagogischen Hochschule Karlsruhe wurde. Die Umstände seiner Berufung nach Karlsruhe sind uns nicht bekannt. Spieler wurde verschiedentlich ausgezeichnet und geehrt.

Während die Arbeiten von Schäper (2006) und Kersting (2008) Spielers Vergangenheit höchstens am Rande diskutieren, zeichnen sie ein ambivalentes Bild des Instituts für Caritaswissenschaften an der Universität Freiburg i. Br., Spielers erster Station nach seiner Rückkehr. Während Professor Josef Beeking die Lehrbefugnis von den Nationalsozialisten im Jahr 1935 entzogen wurde, machte sich dessen Vorgänger Joseph Mayer in einer Abhandlung 1927 für weitreichende staatliche Eingriffe zur „Unfruchtbarmachung Geisteskranker“ stark (Mayer 1927). Mayer trat 1945 aus dem Hochschuldienst aus. Bereits 1934 in den Ruhestand getreten ist der erste Direktor des Instituts, Franz Keller, der sich offen dafür aussprach, die Caritas in den „Dienst an der Rassenhygiene“ zu stellen (Schäper 2006: 173). Bopp wiederum versuchte nach 1933 als „Brückenbauer“ zwischen dem katholischen Denken und dem Nationalsozialismus zu wirken, da er Berührungspunkte sah (Müller 1997: 314-329; 2007). Er ging dann zunehmend auf Distanz zum Nationalsozialismus und hat sich nach dem Krieg anderen Themen zugewandt, ohne die eigene Haltung öffentlich zu reflektieren.

Genauer untersucht werden müsste, inwiefern das Netzwerk um die Caritas die beiden Freiburger Universitäten miteinander verband. Spieler kam auf Empfehlung von Linus Bopp von der Universität Freiburg i. Br. nach Freiburg i. Ü. (Haeberlin 1991: 762). Spieler und Bopp standen in einem freundschaftlichen Verhältnis zueinander (Müller 1997: 293). Bopp gab zuvor in der Schweiz auf Initiative von Bischof Josef Ambühl einen Kurs zur katholischen Heil- 
pädagogik (Wolfisberg 2002: 114). Ambühl wiederum hatte ebenso an der Universität Freiburg i. Br. studiert (Marti 1997: 10) wie Giuseppe Crivelli und Wilhelm Kissling, beides Direktoren des Caritasverbandes Schweiz. Kissling förderte den Aufbau der Heilpädagogik in Freiburg i. Ü. und bot dort auch Vorlesungen zu Caritaswissenschaften an. Freiburg i. Ü. gewährte ausserdem Beeking Zuflucht (Raab 1991: 303). Crivelli hatte sich für eine Berufung des von Bopp beeinflussten Montalta stark gemacht (vgl. Haeberlin 1990: 332; Wolfisberg 2002: 118). Nach bisherigem und lückenhaftem Stand der Forschung scheint Spieler demnach Teil eines katholischen Netzwerks gewesen zu sein, dessen Mitglieder durchaus unterschiedliche Positionen vertreten haben.

\section{Die lückenhafte Aufarbeitung nationalsozialistischer Sympathien an der Universität Freiburg i. ̈.}

Wie sich im Folgenden zeigen wird, ist Josef Spielers Landesverweis auch im Kontext einer lückenhaften innerschweizerischen Aufarbeitung nationalsozialistischer Sympathien zu sehen. Josef Spieler war einer von insgesamt 2318 Personen, welche von der eidgenössischen oder einer kantonalen Exekutive eine Ausweisungsverfügung erhielt (Brassel-Moser 1999: 136). Diese Landesverweisungen aufgrund einer Gefährdung der inneren oder äusseren Sicherheit wurden paradoxerweise erst kurz vor dem Ende des Zweiten Weltkriegs oder danach verfügt (Brassel-Moser 1999: 127). Die Ausweisungen können deshalb als „nachholende[r] Vollzug des Antifaschismus" gesehen werden, der auf deutsche Staatsangehörige kanalisiert wurde (Brassel-Moser 1999: 209-210). Diese Debatten waren untrennbar verwoben mit Auseinandersetzungen um Karriere- und Machtpositionen in der Nachkriegszeit, was einer innerschweizerischen selbstkritischen Auseinandersetzung nicht förderlich war (Brassel-Moser 1999: 213).

Ausgeblendet wurden beispielsweise schweizerische Professoren an der Universität Freiburg i. Ü. mit Sympathien zu Antisemitismus und teilweise Faschismus. Ein Beispiel ist Gonzague de Reynold, der von 1932 bis 1950 einen eigens für ihn geschaffenen Lehrstuhl für die Geschichte der modernen Zivilisation und der Literatur der französischsprachigen Schweiz innehatte. De Reynold bewunderte die katholischen autoritären Regime von Salazar und Mussolini, äusserte sich wohlwollend zu Hitler und vertrat einen säkularen Antisemitismus, dessen Stereotype sich dem von der katholischen Kirche offiziell abgelehnten biologischrassistischen Antisemitismus annäherten (vgl. Altermatt 1999; Prodolliet 2002: 164). Ein weiteres Beispiel ist Jacob Lorenz, von 1935 bis zu seinem Tod 1946 Professor für Soziologie und Wirtschaftskunde. Sein publizistischer Kampf gegen die „Überfremdung“ war offen antisemitisch (Altermatt 1999).

Spielers Professur wurde 1946 mit dem Schweizer Eduard Montalta neu besetzt. Urs Haeberlin stellt Montalta in einem subjektiven Rückblick als „Offizier mit hohem Rang in der Schweizer Armee in eindeutiger Haltung gegen das nationalsozialistische Deutschland“ dar (Haeberlin 2016a: 281). Diese Beschreibung erstaunt angesichts der Tatsache, dass Montalta Spieler öffentlich nie grundsätzlich kritisch hinterfragt hat (Schweizer 2002: 196). Bekannt ist lediglich, dass er in einem Vortrag die nationalsozialistischen Sympathien seines Vorgängers am Rande erwähnt und gleich relativiert hat (Montalta 1983). Dieses öffentliche Schweigen 
Montaltas ist insbesondere auch deshalb bemerkenswert, weil er gemäss seinem Nachfolger Haeberlin darüber informiert gewesen sei, dass Spieler jährlich ins nationalsozialistische Hauptquartier gereist sei und bei einer potenziellen Annexion der Schweiz durch das nationalsozialistische Deutschland als Gau-Leiter vorgesehen gewesen wäre (Haeberlin 2016a: 280; 2016b: 15; 2017: 208).

Für dieses Schweigen Montaltas liefert Haeberlin (2016b: 15) eine Erklärung: Von schweizerischen Katholiken wurde über lange Jahre hinweg behauptet, die Ausweisung von Josef Spieler sein ein ungerechtfertigter Schlag gegen den Katholizismus und die katholische Heilpädagogik gewesen. Eduard Montaltas „eindeutige Haltung gegen den Nationalsozialismus“ scheint also dadurch begrenzt gewesen zu sein, dass er den Erhalt der Integrität der katholischen Verteidigungsstrategie der Aufarbeitung des Falles Spielers vorzog, und dies obwohl er ja anscheinend gewusst haben soll, dass diesem eine hohe Funktion innerhalb der NSDAP zugedacht war.

Auch ein Blick in zwei Werke Montaltas aus dem Jahre 1939 lässt Zweifel aufkommen bezüglich seiner Haltung gegenüber dem nationalsozialistischen Deutschland. In einem Artikel zur nationalsozialistischen Erziehungsidee unterstützt Montalta (1939a) die antiliberalistischen, disziplinorientierten nationalsozialistischen Erziehungsansätze, auch wenn er die „überspitzte Kampferziehung“ und den totalitären Anspruch des nationalsozialistischen Staates ablehnt, weil er den „transzendentalen Beziehungspunkt in der Erziehung“ nicht anerkennt (Montalta 1939a: 532; 524). Die Lektüre dieses Artikels zeigt alles andere als eine eindeutige Haltung das nationalsozialistische Deutschland und bestätigt eher Huonkers (2014: 40) Einschätzung, dass sich Montalta zeitlebens in einem „Netzwerk konservativer wissenschaftlicher Leitfiguren“ "befand und vor Beginn des 2 . Weltkriegs jedenfalls zu Teilen protofaschistisch argumentiert hat. Protofaschismus kann man als eine abgemilderte Form oder Vorstufe des Faschismus verstehen, welche die Wiedergeburt einer ultra-nationalistischen und konservativen Bewegung unterstützt, der aber die taktische und populistische Radikalisierung des Faschismus fehlt (Griffin 2006: 50-51; 241). Auch wenn Montalta zahlreiche Ausprägungen des Nationalsozialismus als zu extrem kritisiert, distanziert er sich nicht von der allgemeinen Stossrichtung der faschistischen Bewegung. Gleich zu Beginn seines Beitrags fragt sich Montalta (1939a: 517), ob der Nationalsozialismus der Beginn einer neuen „Epochenzeit" darstelle und schliesst mit der Feststellung: „voraus ist er uns in der propagandistischen, optimistischen Schlagkraft und in der ungeheuren Zivilcourage der praktischen Verwirklichung“ (Montalta 1939a: 533).

In seiner ebenfalls 1939 erschienenen Schrift „Jugendverwahrlosung“, welche von Josef Spieler herausgegeben wurde, changiert Montalta argumentativ zwischen rassenhygienischer Vererbungslehre und Milieutheorie. So schreibt er zum Beispiel: „Trägt das Milieu die Hauptschuld, so wird in vielen Fällen schon die Versorgung in einer geeigneten Pflegefamilie genügen; kommt aber dazu noch abnorme Anlage, so wird meist Anstaltsversorgung geboten sein“ (Montalta 1939b: 68-69). Diese Argumentation spitzt er explizit antiziganistisch zu. So schreibt Montalta über sogenannte „Vagantenfamilien“: „Ganze Generationen dieser arbeitsscheuen, ruhelosen und nicht seßhaften Elemente leben auf Staatskosten (...) Ein berühmtes Beispiel hat Dr. Jörger in seiner 'Familie Zero' beschrieben. Wenn auch in allen diesen Fällen die ungünstigen Anlagen als Vererbungstatsache eine große Rolle spielen, so muss doch auch 
gesagt werden, daß dazu ebenso mächtige Milieufaktoren sich gesellen“ (Montalta 1939b: 8182). Bemerkenswert ist, dass die vom zitierten Jörger (1905; 1919) verwendete Stammbaumforschung zum damaligen Zeitpunkt dem Stand der wissenschaftlichen Forschung entsprochen hat, aber spätestens 1930 als unwissenschaftlich galt (Kevles 1985) und trotzdem von Montalta zitiert wurde. In seiner Schrift lässt sich Montalta (1939b: 85) über negative Aspekte des Fremdenverkehrs aus, welcher durch das Einführen von ausländischer Literatur in die Schweiz eine negative Auswirkung auf die einheimische Jugend zu haben scheine: „Die Überflutung mit artfremden Elementen (...) hilft mit, schon in unserer Jugend durch Schmuggel von gifttriefender Lektüre (besonders an unseren Mittel- und Hochschulen) den Keim zur Abwendung von jedem Autoritätsgedanken, zur Autoritätslosigkeit und unbeherrschten und unüberlegten Kritisiersucht zu pflanzen.“

Sowohl die beiden diskutierten Publikationen wie auch die trotz gegenteiliger Informationen wenig distanzierte Haltung zu Spieler deuten darauf hin, dass Montalta keineswegs eine eindeutige Haltung gegen das nationalsozialistische Deutschland vertreten hat, sondern eher ein Beispiel für die lückenhafte Aufarbeitung der Vergangenheit im Umfeld der Universität Freiburg i. Ü. darstellt.

\section{Literatur}

Altermatt, Urs (1999). Katholizismus und Antisemitismus: Mentalitäten, Kontinuitäten, Ambivalenzen. Zur Kulturgeschichte der Schweiz, 1918-1945. Frauenfeld: Verlag Huber.

Berger, Manfred (2004). Josef Spieler - Sein Leben und Wirken. heilpaedagogik.de, 2004(2), 25-27.

Berger, Manfred (2010). SPIELER, Karl Jose(ph)f, * 5. August 1900 in Walldürn (Baden), $\uparrow 27$. April 1987 in Karlsruhe, Psychologe, Heilpädagoge, Psychotherapeut, Theologe, Lehrerbildner, Hochschullehrer, Fachpublizist. In: Friedrich Wilhelm Bautz (ursprünglicher Hg.). Biographisch-Bibliographisches Kirchenlexikon. Nordhausen: Verlag Traugott Bautz GmbH, 1255-1268.

Brassel-Moser, Ruedi (1999). Das Schweizerhaus muss sauber sein. Das Kriegsende 1945 im Baselbiet. Liestal: Verlag des Kantons Basel-Landschaft.

Dorand, Jean-Pierre (2017). La Politique Fribourgeoise au 20e siècle. Lausanne: Presses polytechniques et universitaires romandes.

Eberle, Gerhard (2010). Anmerkungen zu einer These Hänsels über das Verhältnis Tornows und Lesemanns während und nach der NS-Zeit. Empirische Sonderpädagogik, 1, 7894.

Englert, Othmar (1942). Die Abnormenzählungen in Deutschland und in der Schweiz unter besonderer Berücksichtigung ihrer pädagogischen und heilpädagogischen Bedeutung. Arbeiten aus dem Heilpädagogischen Seminar der Universität Freiburg (Schweiz), herausgegeben von Dr. Josef Spieler, Heft 13. Luzern: Verlag des Instituts für Heilpädagogik Luzern. 
Griffin, Roger (2006). The Nature of Fascism (3., elektronisch verfügbare Ausgabe). Abingdon: Routledge.

Gügler, Alois (1942). Die erziehliche Behandlung jugendlicher männlicher Onanisten. Arbeiten aus dem Heilpädagogischen Seminar der Universität Freiburg (Schweiz), herausgegeben von Dr. Josef Spieler, Heft 12. O.O.: Selbstverlag des Verfassers.

Haeberlin, Urs (1990). Wie sich Heilpädagogik entwickelt. Illustriert an der Geschichte des Heilpädagogischen Instituts der Universität Freiburg/Schweiz anlässlich deren 100Jahr-Feier. Vierteljahresschrift für Heilpädagogik und ihre Nachbargebiete, 59(1), 327338.

Haeberlin, Urs (1991). Heilpädagogik. In: Roland Ruffieux (Hg.). Histoire de l'Université de Fribourg Suisse 1889-1989, Tome 2: Les Facultés/Geschichte der Universität Freiburg Schweiz, Band 2: Die Fakultäten. Fribourg: Editions Universitaires Fribourg, 762-765.

Haeberlin, Urs (2016a). Ein subjektiv gefärbter Rückblick des scheidenden VHNHerausgebers. Vierteljahresschrift für Heilpädagogik und ihre Nachbargebiete, 85(4), 277-289.

Haeberlin, Urs (2016b). Vergangenheit - Gegenwart - Zukunft. Heilpädagogik im Dilemma zwischen Visionen und Realitäten. In: Verlag des Berufs- und Fachverbandes Heilpädagogik (Hg.). Heilpädagogik. Sinn I Struktur I Perspektiven. Berlin: BHP Verlag, 1124.

Haeberlin, Urs (2017). Inklusive Bildung. Sozialromantische Träumerei? In: Gercke, Magdalena, Saskia Opalinski und Tim Thonagel (Hg.). Inklusive Bildung und gesellschaftliche Exklusion. Zusammenhänge - Widersprüche - Konsequenzen. Wiesbaden: Springer VS, 203-216.

Hänsel, Dagmar (2009). «Erbe und Schicksal». Rezeption eines Sonderschulbuchs. Zeitschrift für Pädagogik, 55(5), 781-795.

Huonker, Thomas (2014). Zum Forschungsstand betreffend Fremdplatzierung in der Schweiz. In: Furrer, Markus, Kevin Heiniger, Thomas Huonker, Sabine Jenzer und AnneFrançoise Praz (Hg.) Fürsorge und Zwang: Fremdplatzierung von Kindern und Jugendlichen in der Schweiz 1850-1980. Basel: Schwabe Verlag, 39-50.

Jörger, Josef (1905). Die Familie Zero. Archiv für Rassen- und Gesellschafts-Biologie, einschliesslich Rassen- und Gesellschafts-Hygiene, 2(4), 494-559.

Jörger, Josef (1919). Psychiatrische Familiengeschichten. Berlin: Julius Springer.

Kevles, Daniel J. (1985). In the Name of Eugenics. Genetics and the Uses of Human Heredity. New York: Alfred A. Knopf.

Kersting, Christa (2008). Pädagogik im Nachkriegsdeutschland. Wissenschaftspolitik und Disziplinentwicklung 1945-1955. Bad Heilbrunn: Verlag Julius Klinkhardt.

Mayer, Joseph (1927). Gesetzliche Unfruchtbarmachung Geisteskranker. Freiburg im Breisgau: Herder \& Co. 
Marti, Christian (1997). Aufbau und Entwicklung des Schweizerischen Caritasverbandes, 1928 bis 1964. Lizentiatsarbeit eingereicht bei der Philosophischen Fakultät. Fribourg: Universität Freiburg $(\mathrm{CH})$.

Montalta, Eduard (1939a). Die nationalsozialistische Erziehungsidee. Monatsschrift des Schweizerischen Studentenvereins, 83. Jahrgang (1938-1939), 517-533.

Montalta, Eduard (1939b). Jugendverwahrlosung. Mit besonderer Berücksichtigung schweizerischer Verhältnisse, eidgenössischer und kantonaler Erlasse. Arbeiten aus dem Heilpädagogischen Seminar der Universität Freiburg (Schweiz), herausgegeben von Dr. Josef Spieler, Heft 8. Zug: Verlag Eberhard Kalt-Zehnder.

Montalta, Eduard (1983). Rückblick auf die Entwicklung der Heilpädagogik in der Schweiz aus der Sicht eines Beteiligten. Vortrag, gehalten am Donnerstag, 24. Februar 1983, 16.1518.00 Uhr, im Auditorium des Heilpädagogischen Instituts der Universität Freiburg, Petrus-Kanisius-Gasse 21, für die Studierenden der Heilpädagogik. Verfügbar auf http://www.montalta.com/em rb.htm (Zugriff am 9.5.2019).

Müller, Philipp (1997). Dem Leben dienen. Das Seelsorgeverständnis von Linus Bopp (18871971) im Kontext heutiger Seelsorgekonzeptionen. Würzburg: Seelsorge Echter.

Müller, Philipp (2007). Die Haltung des Freiburger Pastoraltheologen Linus Bopp (18871971) zum und im Nationalsozialismus. Freiburger Diözesen-Archiv, 127, 135-155.

Oser, Fritz (1991). Pädagogik. In: Ruffieux, Roland (Hg.). Histoire de l'Université de Fribourg Suisse 1889-1989, Tome 2: Les Facultés/Geschichte der Universität Freiburg Schweiz, Band 2: Die Fakultäten. Fribourg: Editions Universitaires Fribourg, 759-762.

Pittet, Faustin (1942). Die Stellung der Kirche zur Eugenik und Rassenhygiene. Arbeiten aus dem Heilpädagogischen Seminar der Universität Freiburg (Schweiz), herausgegeben von Dr. Josef Spieler, Heft 11. Luzern: Verlag des Instituts für Heilpädagogik.

Prodolliet, Simone (2002). Zwischen den Ambivalenzen: Zur Flüchtlingspolitik des Schweizerischen Caritasverbandes während des Zweiten Weltkrieges. In: Caritas (Hg). Von der katholischen Milieuorganisation zum sozialen Hilfswerk. 100 Jahre Caritas Schweiz. Luzern: Caritas-Verlag, 161-173.

Raab, Heribert (1991). Die deutschsprachigen Länder. In: Roland Ruffieux (Hg.). Histoire de l'Université de Fribourg Suisse 1889-1989, Tome 1: Fondation et développement/Geschichte der Universität Freiburg Schweiz, Band 1: Entstehung und Entwicklung. Fribourg: Editions Universitaires Fribourg, 278-307.

Schäper, Sabine (2006). Ökonomisierung in der Behindertenhilfe. Praktisch-theologische Rekonstruktionen und Erkundungen zu den Ambivalenzen eines diakonischen Praxisfeldes. Münster: Lit Verlag. 
Schweizer, Magdalena (2002). Die psychiatrische Eugenik in Deutschland und in der Schweiz zur Zeit des Nationalsozialismus. Ein Beitrag zur geschichtlichen Aufarbeitung mit besonderer Berücksichtigung der Entwicklung der Kinder- und Jugendpsychiatrie in der Schweiz. Bern: Peter Lang.

Tschanz, Christoph und Daniel Künzler (2018). Josef Spieler an der Universität Freiburg i.Ü. (1935-1945/1947). Ergänzung zur Kurzbiographie: „Berger, Manfred (2004). Josef Spieler - Sein Leben und Wirken. heilpaedagogik.de, 2004(2), 25-27.“ Geschrieben für das Internationale Archiv für Heilpädagogik https://archiv-heilpaedagogik.de/neuerbeitrag-josef-spieler-in-freiburg-i-ue/ (Zugriff am 9.5.2019).

Wolfisberg, Carlo (2002). Heilpädagogik und Eugenik. Zur Geschichte der Heilpädagogik in der deutschsprachigen Schweiz (1800-1950). Zürich: Chronos Verlag. 